\title{
Scaling and Integral Solutions to Mixed Convection Over an Exponential Stretching Sheet
}

\author{
Rudra Murthy B. Veerabhadrappa*, Vashist Ademane, Veershetty Gumtapure, Vijay Kumar Hindasageri \\ Department of Mechanical Engineering, National Institute of Technology Karnataka, Surathkal 575025, India
}

Corresponding Author Email: rudra.me16f13@ nitk.edu.in

https://doi.org/10.18280/mmep.070412

Received: 11 August 2020

Accepted: 30 October 2020

\section{Keywords:}

mixed convection flow, heat transfer, similarity

solution, scaling analysis, integral solution

\begin{abstract}
The reported studies on mixed convection flow problems have been solved purely by method of similarity studies. Scaling analysis is an alternate method that can give better engineering insight of the problem being investigated. Integral solutions are mathematically simpler to handle as the engineering requirement is that of accurate solutions only close to the wall. In the present work, scaling and integral solutions are discussed for a typical mixed convection flow problem already discussed in literature by similarity technique. Scaling method has been demonstrated and is found in good agreement with the results obtained from similarity method. The integral solution is obtained by deriving the integral form of governing equation and solution is discussed for specific case of Prandtl number $=1$. The solution obtained by Integral formulations is in good agreement with that of similarity method.
\end{abstract}

\section{INTRODUCTION}

The effect of heat transfer by developing boundary layer of a continuous stretching sheet with respect to a given temperature, moving in a quiescent fluid medium has been more enticed during the last few decades due to its wide applications in many manufacturing processes. The technologies like hot rolling, wire drawing, glass-fiber, and paper production and polymer extrusion and metal spinning. The raw material in red hot condition passed through a different die and extruded to sheet or wires. The stretching thickness and diameter of the wire depend upon the velocity of red-hot raw material moving under the die as well as the pressure applied, on moving red hot raw material. After the desired thickness or diameter obtained, the next set of operation is to cool the hot product to get the desired characteristics. The outcome product feature mainly depends on the factors like heat transfer rate at the continuous stretching surface, exponential changing of stretching velocity and also the temperature distribution. Extruding the wires or thin sheets in a conducting fluid induces a magnetic field. Magneto hydrodynamic fluid (MHD) stabilizes the temperature distribution to maintain the desired characteristics of extruded wire or sheets. Prime importance is the kinematics of stretching, and the process of cooling or heating have a significant effect on the quality of the final product. After the first investigation of Sakiadis [1], several types of research examining the cause of flow induced by a surface moving with fixed velocity [2, 3] have done. Ali [4] investigated the development of a thermal boundary layer based on power-law on the stretched surface with suction and injection. Magyari and Keller [5] carried out their work based on the exponential stretching surface by similarity solutions and compared the results with the power-law model. The effect of radiation on steady MHD boundary layer flow over an exponentially stretching sheet has been carried out by Ishak [6], and found that the heat transfer rate enhances with Prandtl number, but decline with both magnetic parameter and radiation effect. An analysis carried out by Patil et al. [7] to study the unsteady mixed convection flow over a moving vertical surface, considering heat generation or absorption and concluded that unsteadiness is caused due to time-dependent free stream velocity as well as stretching velocity and also buoyancy force enhances the skin-friction coefficient and local Nusselt number.

The MHD fluid discovers applications in many types of stretching sheet issues. However, the study of the magnetic effect on the heat transfer process is more important. Devi and Thiagarajan [8] performed similarity solutions to investigate the flow and heat transfer behavior over a stretching plate under the impact of a non-linear transverse magnetic field. Mukhopadhya $[9,10]$ studied the characteristics of boundary layer flow and heat transfer on a porous exponential stretching surface in the effect of the magnetic field by considering velocity slip and thermal and concluded that horizontal velocity decreases with increasing slip parameter as well as with increasing magnetic effect and temperature increasing with increasing value of the magnetic effect. Costa [11] performed a time scale-based analysis for studying laminar convective phenomena and concluded that the method is very effective to compare the studied situation and obtained momentum and thermal boundary layer thickness.

Pal [12] has been carried out a similarity solution to investigate the mixed convection heat transfer on an exponentially stretching sheet with an exponential temperature distribution under the influence of the magnetic field and internal heat generation or absorption. The application of powerful analytical techniques like scaling and integral approach has not been reported so far. This study deals with the application of scaling and integral approach for mixed convection heat transfer [13] on an exponential stretching surface and the obtained results comparing with similarity solutions reported by $\mathrm{Pal}$ [12]. The phenomenon of laminar convective flow can be solved using analytical methods such 
as scale analysis, similarity analysis and integral analysis. While the similarity solution is purely mathematical, the scale analysis and integral solutions are based on physical reasoning and mathematical approximations [13].

\section{PROBLEM DESCRIPTION}

A two-dimensional steady flow of an electrically conducting with MHD and incompressible viscous fluid over an impermeable vertical surface shown in Figure 1 is stretching with velocity $u_{w}$ varying exponentially with reference velocity $u_{o}$ and a given temperature distribution $\mathrm{T}_{\mathrm{w}}$. The $\mathrm{X}$-axis is considered alongside the elongating of the plane wall and as well as towards the direction of motion. The Yaxis is perpendicular to the $\mathrm{X}$-axis and it is along the direction of applied magnetic field $\mathrm{B}_{0}$. It is assumed that the induced magnetic field of the flow is small in comparison with the applied magnetic field which correlates to negligible magnetic reynolds number.

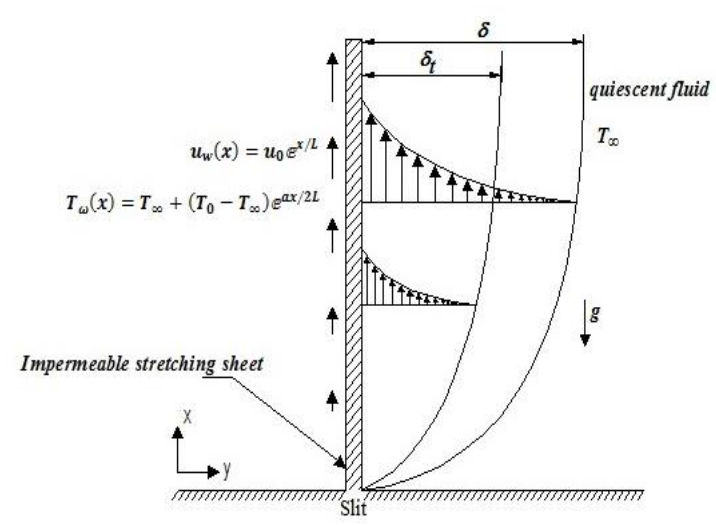

Figure 1. Schematic representation of heat transfer over an exponential stretching sheet with mixed convection boundary condition

The general continuity, energy and momentum equation with the consideration of Boussinesq approximation can be expressed as [12]:

$$
\begin{gathered}
\frac{\partial u}{\partial x}+\frac{\partial v}{\partial y}=0 \\
u \frac{\partial u}{\partial x}+v \frac{\partial u}{\partial y}=v \frac{\partial^{2} u}{\partial y^{2}}+g \beta\left(T-T_{\infty}\right)-\sigma \frac{B_{o}^{2} u}{\rho} \\
u \frac{\partial T}{\partial x}+v \frac{\partial T}{\partial y}=\alpha \frac{\partial^{2} T}{\partial y^{2}}+\frac{\sigma B_{o}^{2} u^{2}}{\rho c_{p}} \\
+\frac{\mu}{\rho c_{p}}\left(\frac{\partial T}{\partial y}\right)^{2}+\frac{Q}{\rho c_{p}}\left(T-T_{\infty}\right)
\end{gathered}
$$

The boundary condition are considered as follows [12]:

$$
\begin{gathered}
u=u_{w}(x), v=0, T=T_{w}(x), \text { at } y=0 \\
u=0, T \rightarrow T_{\infty} \text { as } y \rightarrow \infty
\end{gathered}
$$

The stretching velocity $u_{w}(x)$ and the exponential wall temperature distribution $T_{w}(x)$ are defined as [12]:

$$
u_{w}(x)=u_{0} \mathrm{e}^{x / L}
$$

$$
T_{\omega}(x)=T_{\infty}+\left(T_{0}-T_{\infty}\right) \mathrm{e}^{a x / 2 L}
$$

where ' $T_{o}$ ' and ' $a$ ' denotes the temperature distribution parameters on the exponentially stretching wall.

The parameters are non-dimensionalized as below [12]:

$$
X=\frac{x}{L}, \frac{u_{w}(x)}{u_{0}}=\mathrm{e}^{x / L}=e^{X}, \frac{T_{w}(x)-T_{\infty}}{\left(T_{0}-T_{\infty}\right)}=\mathrm{e}^{a x / 2 \cdot L}=e^{a X / 2}
$$

\section{SCALING ANALYSIS}

Applying Scaling transformation to the Eq. (1) and considering along $x \rightarrow L$ and $y \rightarrow \delta$ or $\delta_{T}$.

$$
\begin{gathered}
\frac{\partial u}{\partial x} \sim \frac{u_{w}(x)}{L} \text { and } \frac{\partial v}{\partial y} \sim \frac{v}{\delta} \\
\frac{u_{w}(x)}{L} \sim \frac{v}{\delta}
\end{gathered}
$$

For two dimensional flows the $\mathrm{x}$ and $\mathrm{y}$ convective time scales are of same order of magnitude. Eq. (9) will be utilized for the flow analysis in the present work.

$$
\frac{u_{0} e^{X}}{L} \sim \frac{v}{\delta} \text { or } \frac{u_{0}}{L} \sim \frac{v}{\delta} e^{-X}
$$

\subsection{Forced Convection}

\subsubsection{Velocity Boundary layer}

Moving magnetic force term to left hand side and neglecting the effect of buoyancy the momentum Eq. (3) can be written as:

$$
u \frac{\partial u}{\partial x}+v \frac{\partial u}{\partial y}+\frac{\sigma B_{0}^{2} u}{\rho}=v \frac{\partial^{2} u}{\partial y^{2}}
$$

Referring to the mass continuity scaling Eq. (9) we conclude that the two inertia term are of same order of magnitude.

$$
\frac{u_{w}^{2}(x)}{L} \sim \frac{v u_{w}(x)}{\delta}
$$

Using scaling rule and scaling transformation Eq. (11) becomes:

$$
\frac{u_{w}^{2}(x)}{L}, \frac{\sigma B_{0}^{2} u_{w}(x)}{\rho} \sim \frac{v u_{w}(x)}{\delta^{2}}
$$

Dividing the Eq. (13) through friction scale $\frac{v u_{w}(x)}{\delta^{2}}$ and using scaling rule, inertia $\sim$ friction force.

$$
\frac{\delta^{2}}{L^{2}} \sim \frac{v}{u_{0} L} e^{-X}
$$




$$
\delta \sim L \operatorname{Re}^{-1 / 2} e^{-X / 2}
$$

where, $R e=\frac{u_{0} L}{v}$ is Reynolds number.

For purely forced convection exponential induced magnetic force as stretching surface passing through electrical conducting quiescent fluid is balanced by frictional force due to viscous shear between stretching surface and quiescent fluid. (Induced magnetic field $\sim$ friction force).

$$
\begin{gathered}
\frac{\sigma B_{0}^{2} u_{0} e^{X}}{\rho} \sim \frac{v u_{0} e^{X}}{\delta^{2}} \\
\delta^{2} \sim \frac{v \rho L^{2}}{\sigma B_{0}^{2} L^{2}} \\
\frac{\delta^{2}}{L^{2}} \sim \frac{v \rho}{\sigma B_{0}^{2} L^{2}} \Rightarrow L\left(\frac{v \rho}{\sigma B_{0}^{2} L^{2}}\right)^{\frac{1}{2}} \\
\delta \sim L H a^{-1}
\end{gathered}
$$

where, $H a=\left(\frac{\sigma B_{0}^{2} L^{2}}{v \rho}\right)^{\frac{1}{2}}$ is Hartmann number.

Comparing the Eq. (15) and Eq. (19):

$$
\begin{gathered}
\left(\frac{H a^{-1}}{R e^{-1 / 2}}\right)^{2} \sim\left(\mathrm{e}^{-X / 2}\right)^{2} \\
\frac{H a^{2}}{R e} \sim \mathrm{e}^{X} \\
\mathrm{M} \sim \mathrm{e}^{\mathrm{X}}
\end{gathered}
$$

where, $M$ is effect of magnetic field:

$$
\mathrm{M}=\frac{H a^{2}}{R e}=\frac{\text { Hartmann number }}{\text { Reynold number }}
$$

From the Figure 1 W.K.T $\delta_{T}<<\delta$ or Pr $>>1$ and it is mixed convection problem. So both natural and forced convection took place.

3.1.2 Thermal boundary formation by forced convection i.e. $\left(\delta_{T}\right)_{\text {F.C }}$

For thin thermal boundary layer $\delta_{T}<<\delta$ of considerably greater scope for fluid in the class of Prandtl number of order 1(example: air) or greater than 1(example: water or oil). Thermal boundary layer thickness is assumed smaller than the velocity boundary layer thickness. Velocity inside the thermal boundary layer is less than stretching velocity of wall surface $u_{w}(x)$.

$$
u \sim u_{w}(x) \cdot \frac{\delta_{T}}{\delta}
$$

Once again referring to the mass continuity, scaling Eq. (9) becomes:

$$
\left(\delta_{T}\right)_{F . C .} \sim \operatorname{LPr}^{-1 / 3} \operatorname{Re}^{-1 / 2} \mathrm{e}^{-X / 2}
$$

$$
\frac{\delta_{T}}{\delta} \sim \operatorname{Pr}^{-1 / 3} \frac{\delta}{\delta_{T}} \sim \operatorname{Pr}^{1 / 3}(\operatorname{Pr} \gg 1)
$$

For $\delta_{T}<<\delta$, the Nusselt number vary as:

$$
N_{u} \sim \operatorname{Pr}^{1 / 3} \operatorname{Re}^{1 / 2} \mathrm{e}^{X / 2}(\operatorname{Pr} \gg 1)
$$

\subsection{Free convection}

3.2.1 Thermal boundary layer formation by natural convection

Consider the governing equations of continuity, momentum, and energy. In the steady-state, the heat conducted from the wall surface into the fluid is swept and move upwards as an enthalpy stream. Eq. (27) signifies a balance between longitudinal convection and transverse conduction (dominant terms for free convection from energy Eq. (3)).

$$
\underbrace{u \frac{\Delta T}{L}, v \frac{\Delta T}{\delta_{T}}}_{\text {convection }} \sim \frac{\alpha \cdot \Delta T}{\delta_{T}^{2}}
$$

where, $\Delta \mathrm{T}=\mathrm{T}_{\mathrm{o}}-\mathrm{T}_{\infty}$ is the scale of the variable $\mathrm{T}-\mathrm{T}_{\infty}$.

In the expression $(14) \mathrm{u}(\Delta \mathrm{T} / L)$ and $\mathrm{v}\left(\Delta \mathrm{T} / \delta_{\mathrm{T}}\right)$ same order of magnitude, by scaling rule [10] we can write it as,

$$
\begin{aligned}
& u \frac{\Delta T}{L} \sim \frac{\alpha \Delta T}{\delta_{T}^{2}} \\
& u_{w}(x) \sim \frac{\alpha L}{\delta_{T}^{2}}
\end{aligned}
$$

By considering Boussinesq approximation and retaining the dominant term for free convection in the momentum Eq. (2). After applying the scaling rule and scaling transformation [13]

$$
\frac{u_{w}^{2}(x)}{L} \sim \underbrace{\frac{v u_{w}(x)}{\delta_{t}^{2}}}_{\text {Friction }} \quad \underbrace{g \beta\left(T_{o}-T_{\infty}\right) e^{a X / 2}}_{\text {Buyoancy }}
$$

Referring to mass conservation scaling (4) signifies that the two inertia terms are of order, $u_{w}^{2}(x) / L$ the buoyancy force is the dominant term because, without it, there would be no free convection. Dividing the expression (30) through the buoyancy scale g. $\beta\left(\mathrm{T}_{0}-\mathrm{T}_{\infty}\right) \mathrm{e}^{a X / 2}$ and substituting Eq. (29) to eliminate vertical velocity scale $u_{w}(x)$, we can write:

$$
\begin{gathered}
\underbrace{\left(\frac{\alpha t}{\delta_{T}^{2}}\right)^{2} \cdot \frac{1}{L^{3}} \cdot \frac{L^{2}}{g \beta\left(T_{o}-T_{\infty} \cdot e^{a X / 2}\right)} \cdot \underbrace{\frac{v \alpha L^{4}}{\delta_{T}^{4}} \cdot e^{X} \cdot \frac{1}{g \beta\left(T_{o}-T_{\infty}\right) \cdot e^{a X / 2} \cdot L^{3}}}_{\text {Friction } / \text { Buoyancy }} \cdot 1}_{\text {Inertia/Buyaancy }} \\
\left(\frac{L}{\delta_{T}}\right)^{4} \cdot e^{a X / 2} \cdot \frac{v}{v} \cdot \frac{\alpha^{2}}{g \beta\left(T_{o}-T_{\infty}\right) L^{3}} \cdot\left(\frac{L}{\delta_{T}}\right)^{4} \cdot \frac{\alpha v e^{-a X / 2}}{g \beta\left(T_{o}-T_{\infty}\right) L^{3}} \cdot 1
\end{gathered}
$$

$$
\left(\frac{L}{\delta_{T}}\right)^{4} R a^{-1} \operatorname{Pr}^{-1} \cdot\left(\frac{L}{\delta_{T}}\right)^{4} R a^{-1} e^{-a X / 2} \cdot 1
$$


where, $R a=\frac{g \beta\left(T_{0}-T_{\infty}\right) L^{3}}{\alpha v}$.

For higher $\operatorname{Pr}$ fluid, i.e., $\operatorname{Pr} \gg 1$.

$$
\begin{gathered}
\left(\delta_{T}\right) \sim L \cdot R a^{-1 / 4} \cdot e^{-a X / 8} \\
u_{w}(x) \sim \frac{\alpha R a^{1 / 2}}{L} e^{a X / 4} \\
N_{u} \sim R a^{1 / 4} e^{a X / 8}
\end{gathered}
$$

The velocity boundary layer $(\delta)$ is driven by a much thinner thermal boundary layer $\left(\delta_{\mathrm{T}}\right)$, and its inertia constrains it. Thus momentum Eq. (2) signifies a balance of inertia and friction force in the layer of thickness $\delta$,

$$
\frac{u_{w}^{2}(x)}{L} \sim \frac{v u_{w}(x)}{\delta^{2}}
$$

After eliminating the vertical velocity scale $u_{w}(x)$ between Eq. (37) and (35) yields

$$
\delta \sim L \operatorname{Ra}^{1 / 4} \operatorname{Pr}^{1 / 2} e^{-a X / 8}
$$

\subsubsection{Mixed convection heat transfer}

If the medium is natural convection, the thermal layer thickness between heat-exchange entities is of order as quiescent fluid provision the buoyant wall jet of thermal boundary $\left(\delta_{T}\right)_{N . C}$ then from Eq. (34).

$$
\left(\delta_{T}\right)_{N . C} \sim L \operatorname{Ra} e^{\frac{-a X}{8}} \operatorname{Pr}>1
$$

On the contrary if the medium is forced convection the wall and fluid reservoir are serrated by a thermal layer thickness of order (Eq. (24)).

$$
\left(\delta_{T}\right)_{F . C} \sim L \operatorname{Pr}^{-1 / 3} \operatorname{Re}^{-1 / 2} e^{-X / 2} \operatorname{Pr}>1
$$

The smaller of the two distances decides the type of convection mechanism. Because the wall dissipate heat to the nearby heat sink (faster mixing), the criterion for transformation from forced to free convection is

If $\left(\delta_{T}\right)_{\text {N.C }}>\left(\delta_{T}\right)_{\text {F.C }}$ governed by natural convection.

If $\left(\delta_{T}\right)_{\text {N.C }}<\left(\delta_{T}\right)_{\text {F.C }}$ governed by forced convection.

For $\operatorname{Pr}>1$

$$
\frac{G r_{1}}{R e^{2}}=\left(\frac{R a^{1 / 4}}{\operatorname{Re}^{1 / 2} \operatorname{Pr}^{1 / 3}}\right)^{4} \operatorname{Pr}^{1 / 3}
$$

where,

$$
G r_{1}=\frac{g \beta\left(T_{0}-T_{\infty}\right) L^{3}}{v^{2}} \text {, i.e. } G r \mathrm{e}^{a X / 2} \mathrm{e}^{-2 X}=\frac{G r_{1}}{R e^{2}}
$$

To establish the Buoyancy effects on a forced convection flow, the main parameter to be considered is the term $\mathrm{Gr}_{1} / \mathrm{Re}^{2}$. This term is essentially the ratio of buoyancy scale to the inertia scale. The buoyancy force arises from the density difference as a result of a temperature gradient in the fluid $G r=\left(G r_{1} \cdot e^{-a x / 2} \cdot e^{2 X}\right) / R_{e}^{2}$. The problem of ascertaining the forced-convection effect on a purely free-convection flow, on the contrary, is found to depend upon the parameter $R e / G r_{1}^{1 / 2}$ [14]. It is worth knowing that the significant governing parameter changes when proceeding from the purely forced convection flow as counter approaching from the purely natural convection flow. For the forced convection process, the parameter is $\mathrm{Gr}_{1} / \mathrm{Re}^{2}$ in contrast for the free convection mechanism, the parameter is $R e / G r_{1}^{1 / 2}$ [14]. The purpose of this scaling approach is to know how the heat transfer and skin friction effected by mixed flow regime.

Applying scaling transformation, we can write energy Eq. (3) as:

$$
\begin{gathered}
u_{w}(x) \frac{\Delta T}{L}, v \frac{\Delta T}{\delta_{T}} \sim \frac{K}{\rho c_{P}} \frac{\Delta T}{\delta_{T}^{2}}, \frac{\sigma B_{0}^{2} u_{w}^{2}(x)}{\rho c_{P}} \\
\frac{\mu}{\rho c_{P}}\left(\frac{\Delta T}{\delta_{T}}\right)^{2}, \frac{Q}{\rho c_{p}}\left(T_{0}-T_{\infty}\right) e^{a X / 2}
\end{gathered}
$$

Claiming flow kinetic energy is balanced by enthalpy difference:

$$
u_{w}(x) \frac{\Delta T}{L} \sim \frac{\sigma B_{0}^{2} u_{w}^{2}(x)}{\rho c_{P}}
$$

Substituting exponential varying of parameters like temperature and velocity with the reference temperature and velocity in the Eq. (42) and simplifying:

$$
\begin{gathered}
\frac{u_{0} e^{X} \Delta T}{L} \sim \frac{\sigma B_{0}^{2} u_{0}^{2} e^{2 X}}{\rho c_{P}}, \frac{u_{0} e^{X}}{L} \sim \frac{\sigma B_{0}^{2} u_{0}^{2} e^{2 X}}{\rho c_{P}\left(T_{o}-T_{\infty}\right) e^{a X / 2}} \\
\frac{u_{0}}{L} \sim \frac{\sigma B_{0}^{2} e^{X} E c}{\rho e^{a X / 2}}
\end{gathered}
$$

where, $E c=\left(u_{0}^{2}\right) /\left(c_{P}\left(T_{0}-T_{\infty}\right)\right)$ is Eckert number, further simplifying the Eq. (44).

$$
E c e^{X} \sim \frac{u_{0} \rho}{\sigma B_{0}^{2} L} e^{a X / 2}
$$

$$
\begin{gathered}
E c e^{X} \sim u_{0} \rho \frac{L}{L} \frac{1}{\sigma B_{0}^{2} L} \frac{v}{v} e^{a X / 2} \\
E c e^{X} \sim \frac{u_{0} L}{v} \frac{v \rho}{\sigma B_{0}^{2} L^{2}} e^{a X / 2}
\end{gathered}
$$

$$
E c e^{X} \sim \frac{R e}{H a^{2}} e^{a x / 2}
$$

$$
e^{a X / 2} \sim E c \frac{H a^{2}}{\operatorname{Re}} e^{X}
$$



(46):

We can substitute the value of $e^{a X / 2}$ from Eq. (8) into Eq.

$$
\frac{T_{\omega}(x)-T_{\infty}}{T_{0}-T_{\infty}} \sim E c \frac{H a^{2}}{R e} e^{x}
$$

The Internal heat generation/absorption by stretching surface lead to a change in enthalpy of quiescent fluid. In the Eq. (41) Claiming internal heat generation/absorption and enthalpy change are of same order of magnitude [13].

$$
\begin{gathered}
\frac{Q}{\rho c_{P}} \Delta T \sim \frac{u_{0} e^{X}}{L} \Delta T \\
e^{X} \sim \frac{Q L^{2}}{\mu c_{p}} \frac{v}{u_{0} L} \\
e^{X}=\frac{Q L^{2}}{\pi c_{p} R e} \\
e^{X} \sim \lambda
\end{gathered}
$$

where, $\lambda=\frac{Q L^{2}}{\pi c_{p} \operatorname{Re}}$ dimensionless heat generation/absorption parameter [15].

By substituting the value of $e^{x}$ from the Eq. (8) into the the Eq. (51), we can write:

$$
\frac{u_{w}(x)}{u_{0}} \sim \lambda
$$

The Eq. (52) signifies heat generation/absorption parameter is a function of stretching velocity of vertical plate. The physical insight of this study is the local skin friction acting on the surface in contact with the ambient fluid of constant density [15], by scaling transformation [13], which can be defined as:

$$
\begin{gathered}
\tau_{w x} \sim \frac{\mu u_{w}(x)}{L} R e_{x}^{1 / 2} \\
\tau_{w x} \sim \rho v \frac{u_{w}(x)}{L} \frac{L}{L} \frac{u_{w}(x)}{u_{w}(x)} R e^{1 / 2}
\end{gathered}
$$

Substituting the Eq. (14) in the Eq. (54) and simplifying:

$$
\tau_{w x} \sim \frac{\rho \delta^{2}}{L^{2}} u_{w}^{2}(x) R e_{x}^{1 / 2}
$$

Now using the Eq. (15) we can further simplify the Eq. (55):

$$
\tau_{w x} \sim \rho u_{w}^{2}(x) R e_{x}^{-1 / 2}
$$

The non-dimensional skin friction coefficient $C_{f}$, can be written as:

$$
C_{f}=2 \frac{\tau_{w x}}{\rho u_{w}^{2}(x)}
$$

Now substituting the Eq. (56) in the Eq. (57) and simplifying by scaling transformation, the new Eq. (58) signifies non-dimensional skin friction coefficient same scale of magnitude of local Reynolds number based on surface velocity.

$$
C_{f} \sim R e_{x}^{-1 / 2}
$$

where, $R e_{x}=\frac{x u_{w(x)}}{v}$.

\section{INTEGRAL SOLUTION}

As a part of this work, an attempt to solve a mixed convection boundary layer flow problem having a constant magnetic field, internal heat generation/absorption, viscous dissipation and buoyancy effect is made. The problem is solved using an integral technique [15]. The thermo-physical properties of the fluid in the flow domain are assumed to be constant except density. The conduction in axial direction is neglected. By applying the first principle the momentum and energy equations can be derived using integral approach.

\subsection{Integral formulation of momentum equation}

Considering an element of dimension $d x \times \delta$ across the boundary layer along $\mathrm{x}$-direction. Figure 2 depicts total forces acting on a differential fluid element.

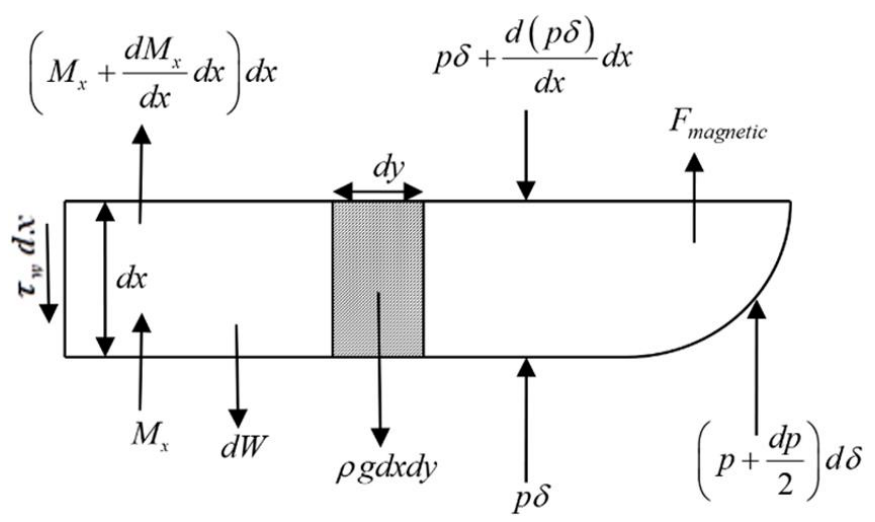

Figure 2. Forces acting on a differential element $d x \times \delta$

The applying Newton's second law to the differential element yields, $\Sigma F_{x}=M_{x}($ out $)-M_{x}($ in $)$.

where,

$\sum F_{x}=$ sum of all the $x$-direction $\left(F_{x}\right)$ forces acting externally on the element

$M_{x}$ (in) = momentum of entering fluid element in $x$-direction $M_{x}$ (out) $=$ momentum of leaving fluid element in $x$-direction Shear stress at the wall,

$$
\tau_{w x}=\mu \frac{\partial u(x, 0)}{\partial y}
$$


Magnetic force on the fluid,

$$
F_{\text {magnetic }}=\frac{\sigma B_{0}^{2}}{\rho} \int_{0}^{\delta} u d y d x
$$

Momentum of entering fluid element in $x$-direction,

$$
M_{x}=\rho \int_{0}^{\delta} u^{2} d y
$$

Weight of the element,

$$
\begin{aligned}
& d W=d x \int_{0}^{\delta} \rho g d y \\
& \left(\begin{array}{l}
p \delta+\left(p+\frac{d p}{2}\right) d \delta-p \delta-\frac{d(p \delta)}{d x} d x-\tau_{0} d x- \\
F_{\text {magnetic }}-d W
\end{array}\right) \\
& =\left(M_{x}+\frac{d M_{x}}{d x} d x\right)-M_{x}
\end{aligned}
$$

By substituting Eq. (59), (60), (61) \& (62) into Eq. (63) gives:

$$
\begin{aligned}
& \left(-\mu \frac{\partial u(x, 0)}{\partial y}-\delta \frac{d p}{d x}-\sigma B_{0}^{2} \int_{0}^{\delta} u d y-\int_{0}^{\delta} \rho g d y\right) \\
& =\rho \frac{d}{d x} \int_{0}^{\delta} u^{2} d y
\end{aligned}
$$

Using Boussinesq approximation for a relation between temperature and density changes, the final integral form of the momentum equation is expressed as follows:

$$
\begin{aligned}
& \left(-v \frac{\partial u(x, 0)}{\partial y}+\beta g \int_{0}^{\delta}\left(T-T_{\infty}\right) d y-\frac{\sigma B_{0}^{2}}{\rho} \int_{0}^{\delta} u d y\right) \\
& =\frac{d}{d x} \int_{0}^{\delta} u^{2} d y
\end{aligned}
$$

\subsection{Integral formulation of energy equation}

The energy balance on the differential element is shown in Figure 3.

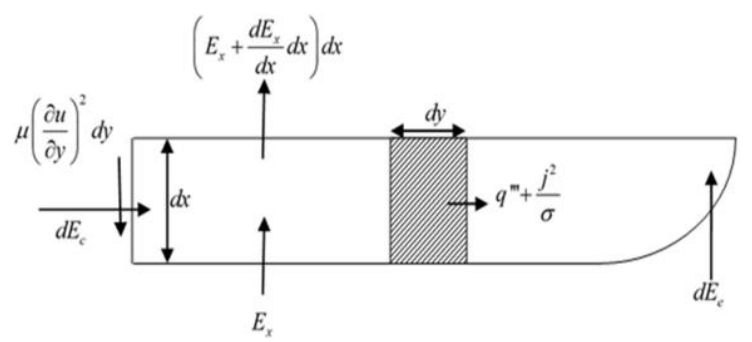

Figure 3. Energy balance on the differential element $\mathrm{dx} \times \delta$

$$
\begin{aligned}
& \left(d E_{c}+d E_{e}+\int_{0}^{\delta_{r}} q^{\prime \prime} d y d x+\int_{0}^{\delta_{1}} \frac{j^{2}}{\sigma} d y d x+\int_{0}^{\delta_{F}}-\mu\left(\frac{\partial u}{\partial y}\right)^{2} d y \mathrm{dx}\right) \\
& =\frac{d E_{x}}{d x} d x
\end{aligned}
$$

where, energy entering the element due to conduction,

$$
d E_{c}=-k \frac{\partial T(x, 0)}{\partial y}
$$

Energy entering the outer edge of the boundary layer,

$$
d E_{e}=C_{p} T_{\infty} \frac{d}{d x} \int_{0}^{\delta_{r}} \rho u \mathrm{dy} \mathrm{dx}
$$

Current density,

$$
j=\sigma B_{0} u
$$

Energy entering the control volume,

$$
E_{x}=\int_{0}^{\delta_{r}} \rho C_{p} u T \mathrm{dy}
$$

Therefore, the integral form of energy equation is

$$
\begin{aligned}
& \left(-\alpha \frac{\partial T(x, 0)}{\partial y}+\int_{0}^{\delta_{r}} \frac{Q\left(T-T_{\infty}\right)}{\rho C_{p}} d y+\frac{\sigma B_{0}^{2}}{\rho C_{p}} \int_{0}^{\delta_{r}} u^{2} d y-\frac{\mu}{\rho C_{p}} \int_{0}^{\delta_{r}}\left(\frac{\partial u}{\partial y}\right)^{2} d y\right) \\
& =\frac{d}{d x} \int_{0}^{\delta_{r}} u\left(T-T_{\infty}\right) d y
\end{aligned}
$$

\subsection{Integral solution}

Consider the problem stated in section scaling part. In this section, both the linear as well as quadratic profiles are considered for both velocity and temperature. In this mixed convection problem, the thermal boundary layer is assumed to be equal to the hydrodynamic boundary layer for simplicity. However, the momentum and energy equations are solved separately.

\subsubsection{Linear velocity and temperature profile}

Boundary conditions considered for a case of linear velocity profile are as follows, $u(x, 0)=U_{w}=U_{0} e^{x / L}, u(x, \delta) \cong 0$.

On simplifying, linear velocity profile is given by:

$$
u=U_{0} e^{x / L}\left(1-\frac{y}{\delta}\right)
$$

Boundary conditions considered for a case of linear temperature profile are as follows:

$$
T(x, 0)=T_{\infty}+\left(T_{0}-T_{\infty}\right) e^{a x / 2 L}, T\left(x, \delta_{T}\right) \cong T_{\infty} .
$$

On simplifying, linear temperature profile is given by:

$$
T=T_{\infty}+\left(T_{0}-T_{\infty}\right) e^{a x / 2 L}\left(1-\frac{y}{\delta_{T}}\right)
$$

Upon substituting the linear temperature and velocity profiles into the Eqns. (64) and (71) and integrating, 


$$
\begin{aligned}
& \left(\frac{v}{\delta} U_{0} e^{x / L}+\beta g\left(T_{0}-T_{\infty}\right) e^{a x / 2 L} \frac{\delta}{2}+\frac{\sigma B_{0}^{2}}{\rho} U_{0} e^{x / L} \frac{\delta}{2}\right) \\
& =\frac{d}{d x}\left(U_{0}^{2} e^{2 x / L} \frac{\delta}{3}\right) \\
& \left(\begin{array}{l}
\left.\frac{\alpha}{\delta_{T}}\left(T_{0}-T_{\infty}\right) e^{a x / 2 L}+\frac{Q\left(T_{0}-T_{\infty}\right)}{\rho C_{p}} e^{a x / 2 L} \frac{\delta_{T}}{2}\right) \\
+\frac{\sigma B_{0}^{2} U_{0}^{2}}{\rho C_{p}} e^{2 x / L} \frac{\delta_{T}}{3}-\frac{\mu U_{0}^{2}}{\rho C_{p}} e^{2 x / L} \frac{1}{\delta_{T}}
\end{array}\right) \\
& =U_{0}\left(T_{0}-T_{\infty}\right) \frac{d}{d x}\left(\frac{\delta_{T}}{3} e^{\frac{x}{L}\left(1+\frac{a}{2}\right)}\right)
\end{aligned}
$$

Required non-dimensional numbers are given as follows:

Hartmann Number, $H a=\left(\frac{\sigma B_{0}^{2} L^{2}}{\mu}\right)^{\frac{1}{2}}$.

Eckert Number, $E c=\frac{U_{0}^{2}}{C p\left(T_{0}-T_{\infty}\right)}$.

Grashoff Number, $G r=\frac{g \beta\left(T_{0}-T_{\infty}\right) L^{3}}{v^{2}}$.

Reynolds Number, $R e=\frac{U_{0} L}{v}$.

Prandtl Number, $\operatorname{Pr}=\frac{v}{\alpha}$.

Dimensionless heat generation or absorption parameter, $\lambda=\frac{Q L^{2}}{\mu C_{p} \operatorname{Re}}$.

Non-dimensional hydrodynamic boundary layer, $\bar{\delta}=\frac{\delta}{L}$.

Non-dimensional thermal boundary layer, $\overline{\delta_{T}}=\frac{\delta_{T}}{L}$.

Dimensional less parameters, $\bar{x}=\frac{x}{L}, \bar{y}=\frac{y}{L}$.

On simplification using Eqns. (74) and (75) and the following ordinary differential equations (ODE) are obtained,

$$
\bar{\delta} \frac{d \bar{\delta}}{d \bar{x}}+\left(2-\frac{3}{2} \frac{H a^{2}}{R e} e^{-\bar{x}}-\frac{3 G r}{2 R e^{2}} e^{(a / 2-2) \bar{x}}\right) \bar{\delta}^{2}=\frac{3}{R e} e^{-\bar{x}}
$$

$\left(\overline{\delta_{T}} \frac{d \overline{\delta_{T}}}{d \bar{x}}+\left(1+\frac{a}{2}-\frac{3}{2} \lambda e^{(a / 2-1) \bar{x}}-\frac{H a^{2} E c}{R e} e^{(1-a / 2) \bar{x}}\right){\overline{\delta_{T}}}^{2}\right)$

$=\left(\frac{3}{\operatorname{Pr} R e} e^{-\bar{x}}-\frac{3 E c}{\operatorname{Re}} e^{(1-a / 2) \bar{x}}\right)$

\subsubsection{Quadratic velocity and temperature profile}

Boundary conditions considered for a case of quadratic velocity profile are as follows:

$$
u(x, 0)=U_{w}=U_{0} e^{x / L}, u(x, \delta) \cong 0, \frac{\partial u(x, \delta)}{\partial y} \cong 0 .
$$

On simplifying, the quadratic velocity profile is given by,

$$
u=U_{0} e^{x / L}\left(1-2 \frac{y}{\delta}+\frac{y^{2}}{\delta^{2}}\right)
$$

Boundary conditions considered for a case of quadratic temperature profile are as follows:

$$
T(x, 0)=T_{\infty}+\left(T_{0}-T_{\infty}\right) e^{a x / 2 L}, T\left(x, \delta_{T}\right) \cong T_{\infty}, \frac{\partial T\left(x, \delta_{T}\right)}{\partial y} \cong 0 .
$$

On simplifying, the quadratic temperature profile is given by,

$$
T=T_{\infty}+\left(T_{0}-T_{\infty}\right) e^{a x / 2 L}\left(1-2 \frac{y}{\delta_{T}}+\frac{y^{2}}{\delta_{T}^{2}}\right)
$$

After substituting the quadratic velocity and temperature profiles into the Eq. (64) and Eq. (71) and integrating,

$$
\begin{aligned}
& \left(\frac{v}{\delta} U_{0} e^{x / L}+\beta g\left(T_{0}-T_{\infty}\right) e^{a x / 2 L} \frac{\delta}{3}+\frac{\sigma B_{0}^{2}}{\rho} U_{0} e^{x / L} \frac{\delta}{3}\right) \\
& =\frac{d}{d x}\left(U_{0}^{2} e^{2 x / L} \frac{\delta}{5}\right) \\
& \left(\begin{array}{l}
\frac{2 \alpha}{\delta_{T}}\left(T_{0}-T_{\infty}\right) e^{a x / 2 L}+\frac{Q\left(T_{0}-T_{\infty}\right)}{\rho C_{p}} e^{a x / 2 L} \frac{\delta_{T}}{3} \\
+\frac{\sigma B_{0}^{2} U_{0}^{2}}{\rho C_{p}} e^{2 x x / L} \frac{\delta_{T}}{5}-\frac{\mu U_{0}^{2}}{\rho C_{p}} e^{2 x / L} \frac{4}{3 \delta_{T}}
\end{array}\right) \\
& =U_{0}\left(T_{0}-T_{\infty}\right) \frac{d}{d x}\left(\frac{\delta_{T}}{5} e^{\frac{x}{L}\left(1+\frac{a}{2}\right)}\right)
\end{aligned}
$$

On further manipulating Eq. (80) and Eq. (81), we obtain the following ODEs in non-dimensional form,

$$
\begin{aligned}
& \bar{\delta} \frac{d \bar{\delta}}{d \bar{x}}+\left(2-\frac{5}{3} \frac{H a^{2}}{\operatorname{Re}} e^{-\bar{x}}-\frac{5 G r}{3} e^{(a / 2-2) \bar{x}}\right) \bar{\delta}^{2}=\frac{10}{R e} e^{-\bar{x}} \\
& \overline{\delta_{T}} \frac{d \overline{\delta_{T}}}{d \bar{x}}+\left(1+\frac{a}{2}-\frac{5}{3} \lambda e^{(a / 2-1) \bar{x}}-\frac{H a^{2} E c}{\operatorname{Re}} e^{(1-a / 2) \bar{x}}\right) \bar{\delta}_{T}^{2} \\
& =\left(\frac{10}{\operatorname{Pr} R e} e^{-\bar{x}}-\frac{4 E c}{\operatorname{Re}} e^{(1-a / 2) \bar{x}}\right)
\end{aligned}
$$

By substituting $\delta^{2}=z$ or $\delta_{T}^{2}=z$, Eqns. (76), (77), (82) and (71) are further simplified into following form:

$$
\frac{d z}{d x}+P z=Q
$$

where, $P$ and $Q$ are functions of ' $x$ '.

The general solution to Eq. (83) is given by:

$$
z \times I . F=\int Q I . F . d x+C
$$

where, I.F $=\mathrm{e}^{\int P \mathrm{dx}}$.

The solution for both non-dimensional hydrodynamic boundary layer and thermal boundary layer over the 
exponentially stretching surface can be expressed as an infinite series. The series solutions are given by:

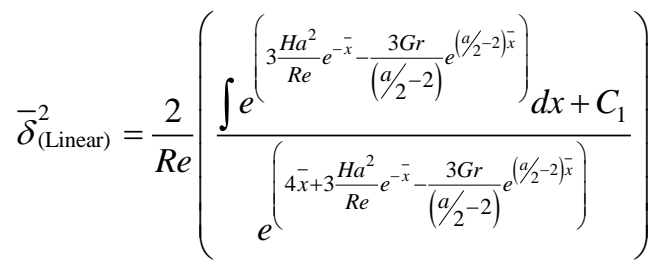

$$
\begin{aligned}
& \bar{\delta}_{\text {(Quadratic) }}^{2}=\frac{20}{3 \operatorname{Re}}\left(\frac{\left.\int e^{\left(\frac{10 H a^{2}}{3 R e} e^{-\bar{x}}-\frac{10 G r}{3(a / 2-2)} e^{(a / 2-2) \bar{x}}\right.}\right)}{e^{\left(4 \bar{x}+\frac{10 H a^{2}}{3 R e} e^{-\bar{x}}-\frac{10 G r}{3(a / 2-2)} e^{(a / 2-2) \bar{x}}\right)}}\right) \\
& \bar{\delta}_{\mathrm{T}(\text { Linear })}^{2}=\frac{\int\left(\frac{6}{\operatorname{Re} P r} e^{-\bar{x}}-\frac{6 E c}{\operatorname{Re}} e^{(1-a / 2)^{\bar{x}}}\right) e^{P} d x+C_{3}}{e^{P}} \\
& \bar{\delta}_{\text {T(Quadratic) }}^{2}=\frac{\int\left(\frac{20}{\operatorname{RePr}} e^{-\bar{x}}-\frac{8 E c}{\operatorname{Re}} e^{(1-a / 2)^{\bar{x}}}\right) e^{q} d x+C_{4}}{e^{q}}
\end{aligned}
$$

where,

$$
q=\left((2+a) \bar{x}-\frac{10}{3\left(\frac{a}{2}-1\right)} \lambda e^{((a / 2)-1) \bar{x}}-\frac{2 H a^{2} E c}{\operatorname{Re}\left(1-\frac{a}{2}\right)} e^{(1-(a / 2))^{\bar{x}}}\right)
$$

The integrations in the Eqns. (86)-(89) have been performed after doing a series expansion of the exponential term/s and the same is computed using a computer code. The integration constants have been evaluated using the following boundary conditions.

$$
\delta(0, y)=0, \delta_{T}(0, y)=0
$$

\section{RESULTS AND DISCUSSION}

\subsection{Scaling analysis}

The following are the comparison of results obtained in the present study (scaling analysis) and similarity solutions reported by Pal [12].

1. Velocity distribution decreases with increasing value of Hartmann number [12].

i.e. Scaling analysis approach predict, similar variation from Eq. (10): $\frac{v}{\delta} \sim \frac{u_{w}}{L}=>\frac{v}{u_{w}} \sim \frac{\delta}{L}=>\frac{v}{u_{w}} \sim H a^{-1}$.

2. Decreases in velocity as $X$ value increment in the boundary layer however, significant nearby the stretching wall [12].

i.e. Scaling analysis approach outcome, the same relation from Eq. (9): $\frac{v}{\delta} \sim \frac{u_{w}}{L}=>\frac{v}{u_{w}} \sim \operatorname{Re}^{-1 / 2} e^{-X / 2}$.

3. The value of Eckert number $\left(E_{c}\right)$ is directly influence to upsurge the temperature in flow region [12].

i.e Scaling analysis approach concludes the same relation from Eq. (47), as Eckert number (Ec) increases the temperature in the flow region increases.

$$
\frac{T_{w}(x)-T_{\infty}}{T_{0}-T_{\infty}} \sim E c \frac{H a^{2}}{R e} e^{X}
$$

4. The thermal boundary layer thickness inverse function of $a, \mathrm{X}$ and $\mathrm{Gr}$ [12].

i.e Scaling analysis approach concludes the same relation from Eq. (34): $\delta_{T} \sim L R a^{-1 / 4} e^{-a X / 8} \sim L\left(G r_{1} P r\right)^{-1 / 4} e^{-a X / 8}$.

5. The effect of Hartmann number $\left(H_{a}\right)$ is direct function of the temperature distribution [12].

i.e Scaling analysis approach concludes the same relation from Eq. (47): $\frac{T_{w}(x)-T_{\infty}}{T_{0}-T_{\infty}} \sim E c \frac{H a^{2}}{R e} e^{X}$.

6. Skin friction coefficient directly proportional to the temperature distribution parameter $(a)$ [12].

Form scaling analysis approach concludes the same relation from Eqns. (46) and (58):

$$
e^{a X / 2} \sim E c \frac{H a^{2}}{R e} e^{X}, C_{f} \sim R e_{x}^{-1 / 2}, \Rightarrow R e_{x}^{-1 / 2} \sim e^{a X / 2}, \therefore C_{f} \sim e^{a X / 2} .
$$

7. The local Nusselt number increases with increment the magnetic field force [12].

From scaling analysis approach concludes the same relation from Eqns. (21) and (26):

$$
N_{u} \sim \operatorname{Pr}^{1 / 3} \operatorname{Re}^{1 / 2} \mathrm{e}^{X / 2}, \frac{H a^{2}}{\operatorname{Re}}=e^{X}, \therefore N u \sim H a \operatorname{Pr}^{1 / 3} .
$$

8. The result of increasing the scale of Prandtl number $(\mathrm{Pr})$ is to increase Nusselt number $(\mathrm{Nu})$ [12].

Scaling analysis approach concludes the same relation from Eq. (26): $N u \sim \operatorname{Pr}^{1 / 3} \operatorname{Re}^{1 / 2} e^{X / 2}$.

\subsection{Integral solution}

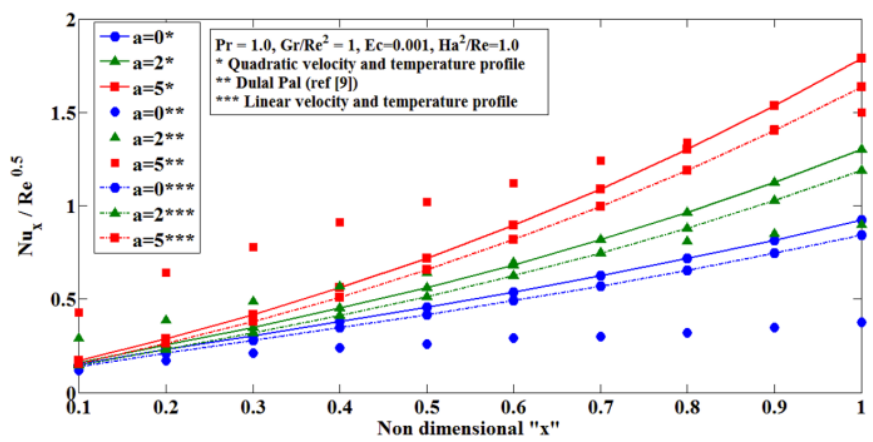

Figure 4. Comparison of local Nusselt number variation along non-dimensional ' $x$ ' with different ' $a$ ' value

An analytical study of the convective heat transfer coefficient for an exponentially continuous stretching surface in a steady flow region with exponential temperature variation at the wall is carried out. The study has been conducted by considering the influence of buoyancy force, magnetic field, internal heat generation/absorption and viscous dissipation effects. The analysis is restricted to $P r=1$. Effect of parameters such as Grashoff number, $\mathrm{Ha}^{2} / \mathrm{Re}$, Eckert number and 'a' on 
local Nusselt number and coefficient of friction (Eq. (66)) over the exponentially stretching surface has been analyzed. A comparative study of the effect of ' $a$ ' on local Nusselt number have been shown in Figure 4.

The Nusselt number obtained from the present integral method and similarity solution results of Dulal Pal [12] are compared in Figure 4. There is an exponential upsurge in the Nusselt number along the non-dimensional distance is observed from the present method. At any ' $x$ ', with the increase in ' $a$ ' shows, there is a heat transfer from the surface to the ambient. The difference in the Nusselt number from the present method with that of Dulal Pal [12] can be attributed to the solution method used in the corresponding study.

$$
C_{f}=\frac{\tau_{w x}}{\frac{1}{2} \rho U_{w}^{2}}=\frac{-4}{\operatorname{Re}_{L}} \frac{e^{-\bar{x}}}{\bar{\delta}}
$$

Skin friction coefficient results presented in Table 1 shows better agreement with the results of Dulal Pal [12] for all ' $a$ ' value. Quadratic velocity and temperature profile assumption result in less error in the calculation of skin friction coefficient that the linear profile assumption.

Table 1. Comparison of coefficient of friction variation with ' $a$ ' at $P r=1, G r=0.5 \times R e^{2}, H a=0.2$, and $\bar{x}=0.5$

\begin{tabular}{cccccc}
\hline \multirow{2}{*}{$\boldsymbol{a}$} & \multicolumn{3}{c}{$\boldsymbol{C}_{\boldsymbol{f}} \times \boldsymbol{R} \boldsymbol{e}^{\mathbf{0 . 5}}$} & \multicolumn{2}{c}{$\%$ Error } \\
\cline { 2 - 6 } & Pal [9] & Quadratic profile & Linear profile & Quadratic profile & Linear profile \\
\hline-1.5 & -1.14 & -1.19 & -1.10 & 4.31 & -3.24 \\
-1.1 & -1.16 & -1.18 & -1.09 & 1.69 & -5.95 \\
-0.5 & -1.17 & -1.16 & -1.08 & -0.75 & -8.38 \\
0.0 & -1.17 & -1.14 & -1.07 & -2.24 & -9.80 \\
1.0 & -1.16 & -1.11 & -1.03 & -4.46 & -11.74 \\
\hline
\end{tabular}

\section{CONCLUSION}

Scaling and Integral solutions have been applied to the problem of flow over a flat vertical plate with mixed convection boundary condition. It has been demonstrated via scaling that the mathematical rigor required is less and also gives better engineering insight into the problem. The solution obtained by scaling analysis is in good agreement with that of similarity method reported in the literature. Integral formulation of the mixed convection problem is derived and the solution is obtained for a specific case of $\operatorname{Pr}=1$. Perturbation analysis was used to get solution of this problem. The results obtained from the integral solution are in good agreement with that of similarity solution. The advantage of using the integral solution is the reduction in mathematical rigor required to solve the problem.

\section{REFERENCES}

[1] Sakiadis, B.C. (1961). Boundary-layer behavior on continuous solid surfaces: I. Boundary-layer equations for two-dimensional and axisymmetric flow. AIChE Journal, 7(1): 26-28. https://doi.org/10.1002/aic.690070108

[2] Afzal, N. (2010). Momentum and thermal boundary layers over a two-dimensional or axisymmetric nonlinear stretching surface in a stationary fluid. International journal of heat and mass transfer, 53(1-3): 540-547.

http://dx.doi.org/10.1016/j.ijheatmasstransfer.2009.04.0 39

[3] Mabood, F., Khan, W.A., Ismail, A.M. (2017). MHD flow over exponential radiating stretching sheet using the homotopy analysis method. Journal of King Saud University-Engineering Sciences, 29(1): 68-74. https://doi.org/10.1016/j.jksues.2014.06.001

[4] Ali, M.E. (1995). On thermal boundary layer on a powerlaw stretched surface with suction or injection. International Journal of Heat and Fluid Flow, 16(4): 280 290. https://doi.org/10.1016/0142-727X(95)00001-7
[5] Magyari, E., Keller, B. (1999). Heat and mass transfer in the boundary layers on an exponentially stretching continuous surface. Journal of Physics D: Applied Physics, 32(5): 577-585. https://doi.org/10.1088/0022$3727 / 32 / 5 / 012$

[6] Ishak, A. (2011). MHD boundary layer flow due to an exponentially stretching sheet with radiation effect. Sains Malaysiana, 40(4): 391-395.

[7] Patil, P.M., Anilkumar, D., Roy, S. (2013). Unsteady thermal radiation mixed convection flow from a moving vertical plate in a parallel free stream: Effect of Newtonian heating. International Journal of Heat and Mass Transfer, 62: 534-540. https://doi.org/10.1016/j.ijheatmasstransfer.2013.03.027

[8] Devi, S.A., Thiyagarajan, M. (2006). Steady nonlinear hydromagnetic flow and heat transfer over a stretching surface of variable temperature. Heat and Mass Transfer, 42(8): 671-677. https://doi.org/10.1007/s00231-0050640-y

[9] Mukhopadhyay, S. (2013). Slip effects on MHD boundary layer flow over an exponentially stretching sheet with suction/blowing and thermal radiation. Ain Shams Engineering Journal, 4(3): 485-491. https://doi.org/10.1016/j.asej.2012.10.007

[10] Jain, S., Choudhary, R. (2015). Effects of MHD on boundary layer flow in a porous medium due to exponentially shrinking sheet with slip. Procedia Engineering, 127: 1203-1210. http://dx.doi.org/10.1016/j.proeng.2015.11.464

[11] Costa, V.A.F. (2002). A time scale-based analysis of the laminar convective phenomena. International Journal of Thermal Sciences, 41(12): 1131-1140. https://doi.org/10.1016/S1290-0729(02)01399-6

[12] Pal, D. (2010). Mixed convection heat transfer in the boundary layers on an exponentially stretching surface with magnetic field. Applied Mathematics and Computation, 217(6): 2356-2369. https://doi.org/10.1016/j.amc.2010.07.035

[13] Bejan, A. (2013). Convection Heat Transfer. John Wiley $\&$ Sons.

[14] Szewczyk, A.A. (1964). Combined forced and free- 
convection laminar flow. Journal of Heat Transfer, 86(4): 501-507. https://doi.org/10.1115/1.3688729

[15] Jiji, L.M. (2006). Heat Convection. Berlin: Springer. http://dx.doi.org/10.1007/978-3-642-02971-4

\section{NOMENCLATURE}

a wall

$B_{0} \quad$ Applied magnetic field perpendicular to the sheet

$C_{f} \quad$ Local skin friction coefficient

$c_{p} \quad$ Specific heat of fluid

Ec Eckert number

Gr Grashoff number

$g$ Gravitational acceleration

$\mathrm{Ha}$ Hartmann number

$L \quad$ Characteristic length of the vertical sheet

$\mathrm{Nu}$ Nusselt number

Pr Prandtl number

$Q \quad$ Internal heat generation or absorption

$R e_{x}$ Local Reynolds number

$T \quad$ Fluid temperature

$T_{\infty} \quad$ Ambient temperature

$T_{0} \quad$ Reference temperature

$T_{w} \quad$ Wall temperature

$u \quad$ Fluid axial velocity

$u_{0} \quad$ Reference velocity

$u_{w} \quad$ Vertical surface velocity
$V \quad$ Fluid transverse velocity

$X$ Non-dimensional co-ordinate along the sheet

$x, y \quad \begin{aligned} & \text { Co-ordinate } \\ & \text { respectively }\end{aligned}$

\section{Greek symbols}

$\alpha \quad$ Thermal diffusivity

$\beta \quad$ Thermal expansion coefficient

$\delta \quad$ Hydrodynamic boundary layer thickness

$\delta_{T} \quad$ Thermal boundary layer thickness

$\bar{\delta}$ Non-dimensional hydrodynamic boundary layer thickness

$\overline{\delta_{T}} \quad$ Non-dimensional thermal boundary layer thickness

Non-dimensional heat generation or absorption parameter

$\mu \quad$ Fluid viscosity

$v \quad$ Kinematic viscosity

$\rho \quad$ Fluid density

$\tau_{w x}$ Local shear stress

$\sigma \quad$ Electrical conductivity of fluid

\section{Subscripts}

$x \quad$ Local

$w \quad$ Condition on stretching surface

$o$ Reference

$\infty$ Ambient condition 